\title{
Japanese Encephalitis Virus wild strain infection suppresses dendritic cells maturation and function, and causes the expansion of regulatory $T$ cells
}

\author{
Shengbo Cao ${ }^{1,2+}$, Yaoming $\mathrm{Li}^{1,2+}$, Jing Ye ${ }^{1,2}$, Xiaohong Yang ${ }^{1,2}$, Long Chen ${ }^{1,2}$, Xueqin Liu ${ }^{1,3}$, Huanchun Chen ${ }^{1,2^{*}}$
}

\begin{abstract}
Background: Japanese encephalitis (JE) caused by Japanese encephalitis virus (JEV) accounts for acute illness and death. However, few studies have been conducted to unveil the potential pathogenesis mechanism of JEV. Dendritic cells (DCs) are the most prominent antigen-presenting cells (APCs) which induce dual humoral and cellular responses. Thus, the investigation of the interaction between JEV and DCs may be helpful for resolving the mechanism of viral escape from immune surveillance and JE pathogenesis.

Results: We examined the alterations of phenotype and function of DCs including bone marrow-derived DCs (bmDCs) in vitro and spleen-derived DCs (spDCs) in vivo due to JEV P3 wild strain infection. Our results showed that JEV P3 infected DCs in vitro and in vivo. The viral infection inhibited the expression of cell maturation surface markers (CD40, CD80 and CD83) and $\mathrm{MHCl}$, and impaired the ability of P3-infected DCs for activating allogeneic naive $T$ cells. In addition, P3 infection suppressed the expression of interferon (IFN)- $\alpha$ and tumor necrosis factor (TNF)- $\alpha$ but enhanced the production of chemokine (C-C motif) ligand 2 (CCL2) and interleukin (IL)-10 of DCs. The infected DCs expanded the population of CD4+ Foxp3+ regulatory T cell (Treg).

Conclusion: JEV P3 infection of DCs impaired cell maturation and T cell activation, modulated cytokine productions and expanded regulatory T cells, suggesting a possible mechanism of JE development.
\end{abstract}

\section{Background}

JEV is a causative agent of JE which causes at least 50,000 clinical cases and about 10,000 deaths each year. It is a member of the mosquito-borne encephalitis complex of the Flaviviridae family and has recently been discovered in previously non-affected areas like Australia [1] and Pakistan [2]. The neurons in the central nervous system (CNS) are target cells of JEV. Studies show that a direct viral cytopathic response and both direct and indirect immunological responses can contribute to CNS degeneration through JEV-infected cell exclusion by macrophages and CTLs, secretion of cytokines and chemokines and activation of microglia [3-6]. However, few studies have investigated the mechanisms by which JEV evades the immune surveillance of the host and

\footnotetext{
* Correspondence: chenhch@mail.hzau.edu.cn

† Contributed equally

${ }^{1}$ State Key Laboratory of Agricultural Microbiology, Huazhong Agricultural University, Wuhan, Hubei 430070, PR China

Full list of author information is available at the end of the article
}

passes through the blood-brain barrier $(\mathrm{BBB})$ to the CNS.

Dendritic cells (DCs) are the most prominent antigenpresenting cells (APCs) which induce dual humoral and cellular responses. While DCs also play unique role in inducing immune tolerance, avoiding immune surveillance and causing persistent infection. There are studies about the interaction between virus and DCs which showed that viral infection of DCs inhibited the cell maturation and impaired the cell function [7-9]. Human cytomegalovirus (HCMV) infection de-regulated the expression of surface MHC classI, CD40, CD80 and CD86 molecules on DCs. Furthermore, both T cell proliferation and cytotoxicity of $\mathrm{T}$ cells specific to an antigen presented by DCs were reduced via the release of soluble CD83 when DCs were infected with HCMV $[8,10,11]$. Likewise, human immunodeficiency virus (HIV) affected maturation of DCs within the thymus, which contributed to the loss of the naive $\mathrm{T}$ cell and 
memory $\mathrm{T}$ cell population and even facilitated the dissemination of HIV [12].

Additionally, recent studies revealed that several viruses belonging to the Flaviviridae family, such as classical swine fever virus (CSFV), Dengue virus (DV) and Yellow fever virus (YFV), infected DCs and altered the cell phenotype and function [13-15]. Furthermore, Aleyas et al. [2009] recently reported that JEV Beijing strain replicated both in bmDCs and macrophages, and induced functional impairment of DCs through MyD88dependent and independent pathways which subsequently led to poor $\mathrm{CD} 4+$ and $\mathrm{CD} 8+\mathrm{T}$ cell responses [16]. Thus, the investigation of the interaction between virus and DCs is imperative for resolving the viral escape from immune surveillance and JE pathogenesis.

Since there is no evidence for JEV infection of DCs in vivo, we investigated the alteration of phenotype and function of the JEV P3-infected DCs both in vitro and in vivo. Our results indicated that JEV P3 severely infected DCs in vitro and in vivo, and the infection with JEV impaired cell maturation and the capacity for $\mathrm{T}$ cell activation. In addition, our study also showed that the infection of DCs with P3 expanded the population of CD4+ Foxp3+ regulatory T cell (Treg) with immunosuppressive potential, suggesting that the virus-induced alteration of DCs is a likely cause of the immunosuppression found in JEV infection.

\section{Results}

\section{JEV P3 infection of DCs in vitro and in vivo}

The purity of the bmDCs fraction from cell culture or infected mouse splenocytes was higher than $90 \%$ as determined by FACS analysis with surface molecules expression (CD11c). After JEV infection, a 467-bp specific RNA fragment of JEV was detected by RT-PCR (Figure 1A) and the E protein of the JEV was detected by Western blotting in DCs (Figure 1B). FACS results showed over $80 \%$ bmDCs and $90 \%$ spDCs were infected by JEV P3 (Figure 1C). Analysis by real-time PCR showed that DCs supported JEV replication and yielded infectious virus (Figure 1D). These results suggest that JEV infected DCs both in vitro and in vivo.

\section{P3 infection suppressed the maturation of DCs}

DCs present antigen to and activate $\mathrm{T}$ lymphocytes through up-regulating the expression of costimulatory and antigen presentation-associtated molecules at the mature stage [17]. To examine whether the characteristics of immature DCs were altered by P3 infection, we tested the surface molecules of the infected DCs in vitro and in vivo. The expression of maturation surface markers, including CD40, CD80, CD83 and MHClwas upregulated in UV-P3-stimulated, but not in P3-infected bmDCs and spDCs or mock-treated DCs (Figure 2), indicating that UV-P3 stimulation accelerated the maturity of DCs whereas P3 infection dramatically inhibited the cell maturation process.

\section{P3 infection modulated cytokine production of DCs}

In many cases, virus does not directly result in the destruction of host organism but instead causes indirect damage through the disordered release of cytokines [18]. In addition, imbalanced levels of cytokines may contribute to viral persistence and irreversible immunsuppression. Therefore, we examined the profiles of pro- and anti-inflammatory cytokines produced by P3-infected DCs in vitro and in vivo. Our results showed that P3 infection enhanced the releases of IL-10 and CCL2 of DCs but suppressed the production of IFN- $\alpha$ and TNF- $\alpha$ (Figure 3). And it was interesting to show that JEV which was inactivated by UV irradiation failed to induce the production of IL-10 and CCL2 but succeeded in inducing the expression of IFN- $\alpha$ and TNF- $\alpha$. This indicates that the release of CCL2 and IL-10 from DCs was dependent on viral replication, while the production of IFN- $\alpha$ and TNF- $\alpha$ was independent on viral replication.

\section{DCs infected with P3 attenuated allostimulatory activities to $\mathrm{T}$ cells}

To test whether P3 infection will impair the ability of DCs to activate allogeneic naïve $T$ cells, the direct effect of P3-infected DCs in activation of naïve T cells was analyzed by mixed lymphocyte reaction (MLR) and ELISPOT assay. In MLR, the allo-stimulative capability of DCs was significantly suppressed by P3 infection compared to the UV-P3-stimulated group $(P<0.05)$. In addition, the viral infection blocked the LPS-induced allostimulatory activity of DCs (Figure 4A, B).

In ELISPOT assay detecting IFN- $\gamma$ producing $\mathrm{T}$ cells, the number of spot forming units $/ 10^{6}$ purified $\mathrm{T}$ cells was counted after twenty four hour incubation with differently treated bmDCs or spDCs. The results in vitro showed that P3-infected bmDCs activated $25 \pm 9$ spots/ $10^{6}$, while the UV-P3-stimulated bmDCs activated $68 \pm$ 21 naïve $\mathrm{T}$ cells $/ 10^{6}$. In vivo, P3-infected spDCs produced $52 \pm 12$ spots $/ 10^{6}$ whereas UV-P3-stimulated spDCs produced $107 \pm 34$ spots $/ 10^{6}$. This was consistent with the result of MLR assay. P3 infection, in vivo or in vitro, significantly suppressed the ability of DCs to activate allogeneic naïve $\mathrm{T}$ cells in response to LPS treatment (Figure 5A, B and 5C). It implied that P3 infection played an important role in the dysfunction of DCs in activating allogeneic $\mathrm{T}$ cells.

\section{P3-infected DCs expanded Treg}

The immune response may be limited in magnitude and efficacy when the host with normal Treg function is infected with virus. We examined whether P3-infected 
(A)

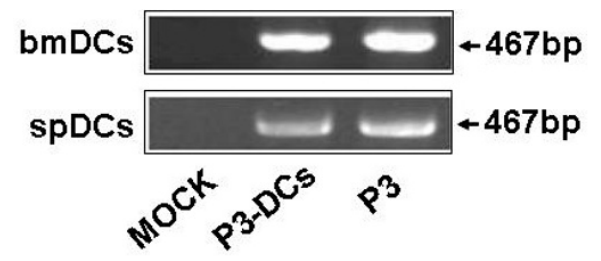

(B)

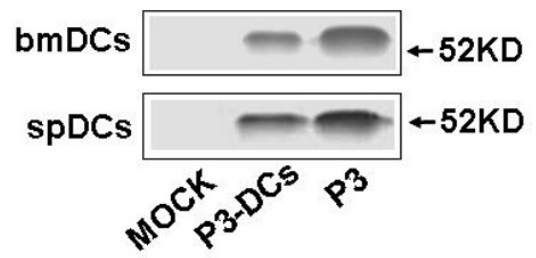

(C)

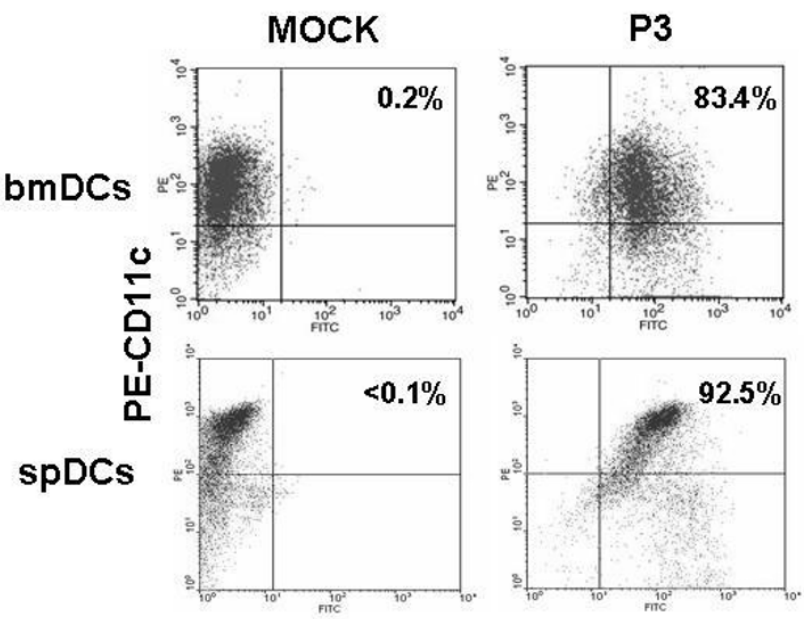

FITC-E

(D)

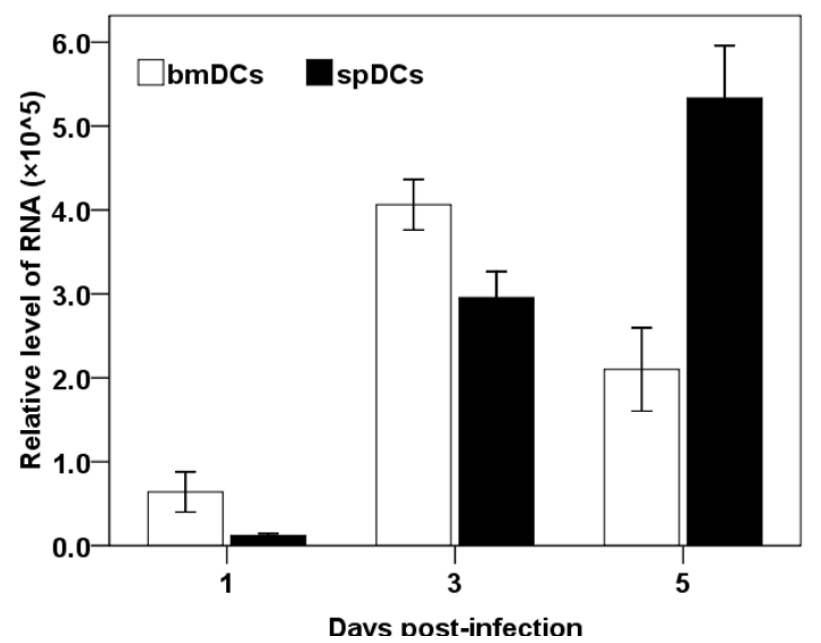

Figure 1 P3 infects DCs in vitro and in vivo. (A) The in vitro infected bmDCs and the spDCs from P3-challenged mice were harvested and analyzed with RT-PCR. Bands shown are 467-bp PCR products specific for JEV. (B) The bmDCs and spDCs were analyzed for E protein (JEV envelope protein) by separation of the proteins on a 10\% SDS-PAGE gel followed by electrotransfer to NC membranes and incubation with monoclonal antibodies against E protein. (C) The bmDCs were harvested after 3 days infection and the spDCs were isolated from mice which had been challenged for 5 days. $1 \times 10^{5}$ bmDCs or spDCs were doubly stained with FITC-anti-E and PE-anti-CD11c and analyzed by FACS respectively. (D) The infected bmDCs and the spDCs from challenged mice were collected 3 times at day 1, 3 and 5, and a real-time PCR was performed to quantitatively detect RNA copies of JEV. Each point represents the mean \pm SD determinants in triplicate. 


\section{(A)}


(C)
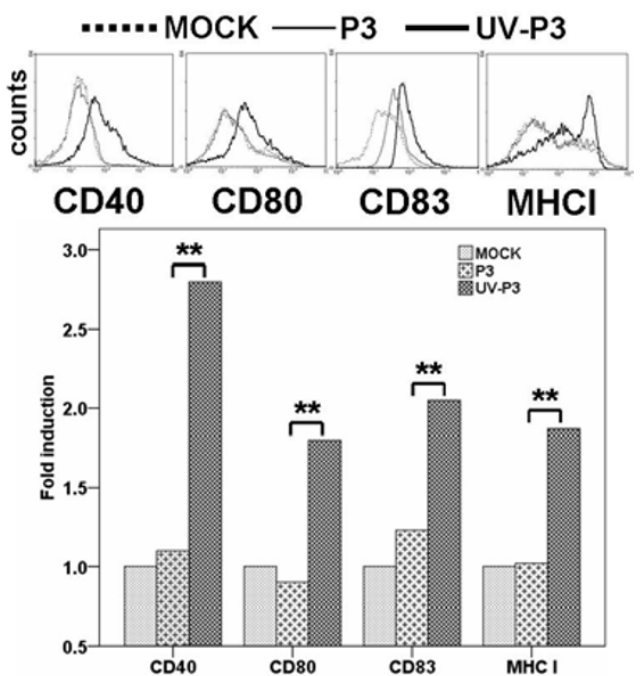

(B)
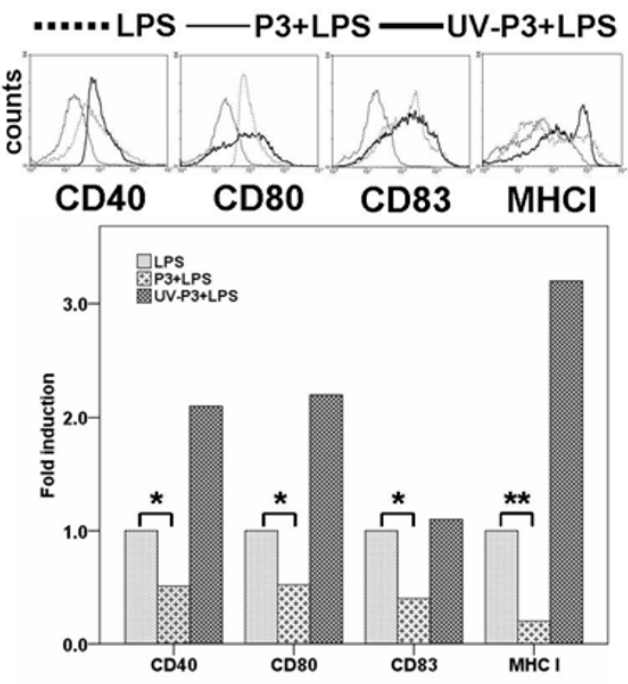

(D)
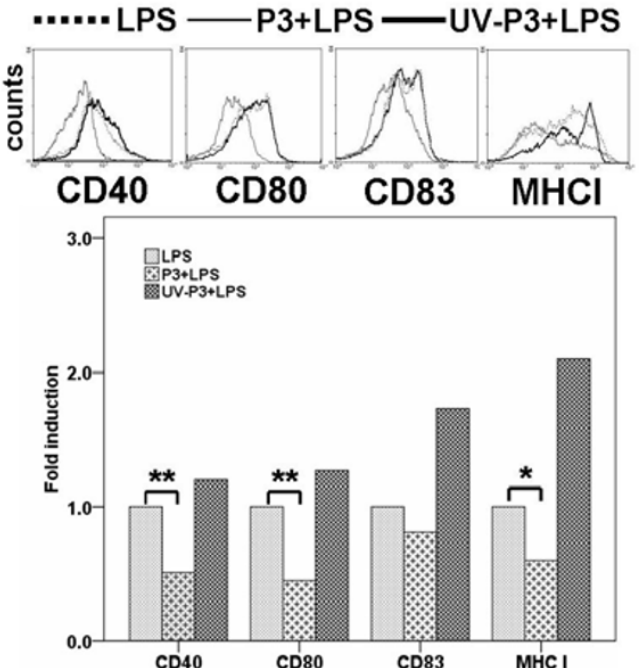

Figure 2 Effects of P3 infection on DCs maturation. $1 \times 10^{5}$ freshly purified bmDCs were left mock-treated or treated with $1 \mathrm{MOI}$ of $\mathrm{P} 3$ or UV-P3 with or without LPS (lipopolysacchide, Sigma-Aldrich, MO) for 3 days. The spDCs from mice, which have been challenged or immunized for 5 days, were obtained and treated with or without LPS. Expressions of CD40, CD80, CD83 and MHCl of the bmDCs (A,B) or spDCs (C,D) were evaluated by FACS. Relative fluorescence intensity to mock group (fold induction) was expressed as the means \pm SD of triplicates. ${ }^{*}, P<0.05$; ${ }^{* *}$, $P<0.01$.

DCs would modulate Treg differentiation. The test revealed that P3-infected bmDCs significantly enhanced the differentiation of Foxp3+ Treg in vitro which was consistent with the results in vivo (Figure 6A, B and 6C). However, the UV-P3-stimulated DCs did not alter the expansion of the Treg, as well as the mock-treated DCs.

\section{Discussion}

Most studies conducted to evaluate the pathogenesis of JEV infection have noted the interaction of the virus with macrophages, microglia and astrocytes, which are major contributors to the production of inflammatory cytokines and CNS degeneration $[3,4,6]$. In the present 


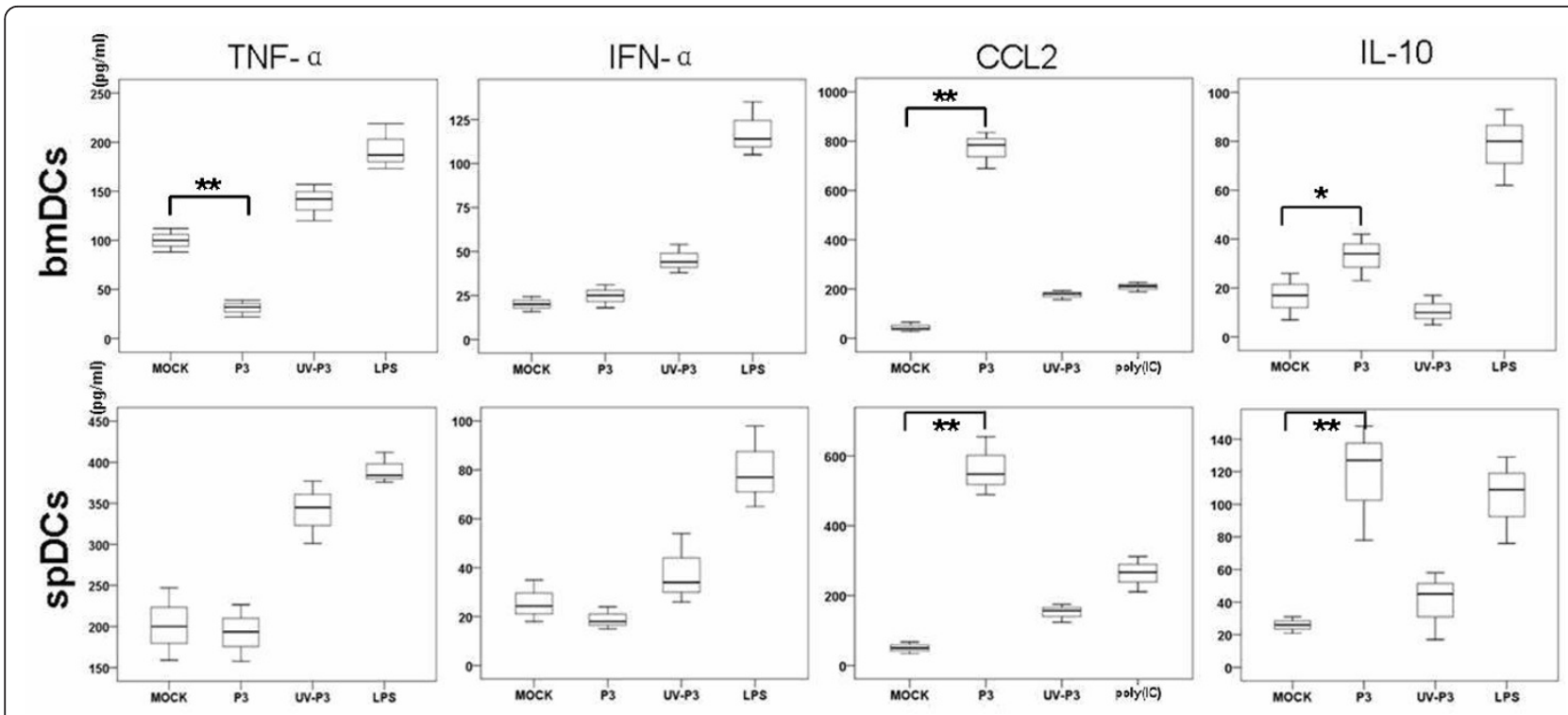

Figure 3 Cytokine profiles of P3-infected DCs (IFN- $\boldsymbol{\alpha}$, TNF- $\boldsymbol{\alpha}$, CCL2 and IL-10). $1 \times 10^{5}$ freshly purified bmDCs were left mock-treated or treated with $1 \mathrm{MOI}$ of P3 or UV-P3 for 3 days. The spDCs from mice, which were challenged or immunized for 5 days, were obtained and cultured for 3 days. The cell supernatants harvested at 3 days of post infection were analyzed with ELISA to measure the concentrations of cytokines (IFN- $\alpha$, TNF- $\alpha$, CCL2 and IL-10). Cytokine concentrations were expressed as the means \pm SD of triplicates. ${ }^{*}, P<0.05 ;{ }^{* *}, P<0.01$.

study, we attempted to address the possible pathogenesis of JEV wild strain infection by testing the interaction of JEV and DCs in vivo and in vitro.

Carrasco et al., [2004] discovered that CSFV could infect and replicate in monocyte and myeloid-derived DCs [14]. Therefore, we hypothesized that JEV, which also belongs to the Flaviviridae family, may affect DCs to facilitate viral spread by escaping immune surveillance. Although Aleyas et al. [2009] recently reported JEV infection of DCs in vitro, whether JEV infects DCs in vivo remained unknown until now. Our research not only verified the results of Aleyas [16], but also investigated the JEV infection of DCs in vivo. Additionally, one of our preliminary experiments showed that when BALB/c mice were inoculated with C6FeK4N6-labeled P3-infected bmDCs or spDCs via intraperitoneal (i.p.), JEV and C6FeK4N6-labeled DCs were detected simultaneouly in the brain of mice with severe symptoms of immunohistochemistry (unpublished data). It is likely that JEV could use DCs as a virus delivery vehicle as it moves through the CNS.

The impaired surface molecule expression of APCs may directly affect the process of antigen presentation and $\mathrm{T}$ cell activation. Thus, we analyzed the alteration of the surface-molecule expression of infected DCs in vitro and in vivo. The FACS analyses revealed an suppressed expression of surface molecules, such as CD40, CD80, CD83 and MHCI, on P3-infected DCs in vitro and in vivo, which is in accordance with Aleyas's results [16]. While we also discovered that the antigen presenting- associated molecules on bmDCs were significantly enhanced after JEV SA14-14-2 strain (a successful JEV live vaccine strain) infection [19]. This suggests the potential molecular mechanism of the immune escape of P3 and the high immunopotency of SA14-14-2.

Since we have verified that JEV infection impaired the expression of antigen presenting-molecules and co-stimulator molecules, whether this impairment of the crucial components on DCs would affect their capacity to activate CD4+ and CD8+ T cell directly is needed to be investigated[20,21]. Thus, we analyzed the capacity of the infected DCs for activating allogeneic $\mathrm{T}$ cells by MLR and ELISPOT assay. It was observed that the T cell activating ability of was dramatically impaired by P3-infection, but boosted by UV-P3 stimulation and SA14-14-2 infection. It has been reported that Hepatitis $\mathrm{C}$ virus (HCV), Ebola viruses and HIV escaped immune surveillance during acute or chronic infection because of the defect of APCs function for activating T cell [21-23]. Therefore it suggested that the impairment of activating of allogeneic naïve T cells of P3 infected DCs could be involved in the JE development.

Treg is a subset of CD4+ T-cell with regulatory properties. Previous studies on the role of Tregs in viral infections suggest that they suppresses antiviral effector $\mathrm{T}$ cell responses or local immune activation at the sites of viral replication [24,25], which may subsequently result in viral immune evasion and the establishment of chronic infections [26-28]. Our FACS results showed that P3 infection contributed to the differentiation of 
(A)
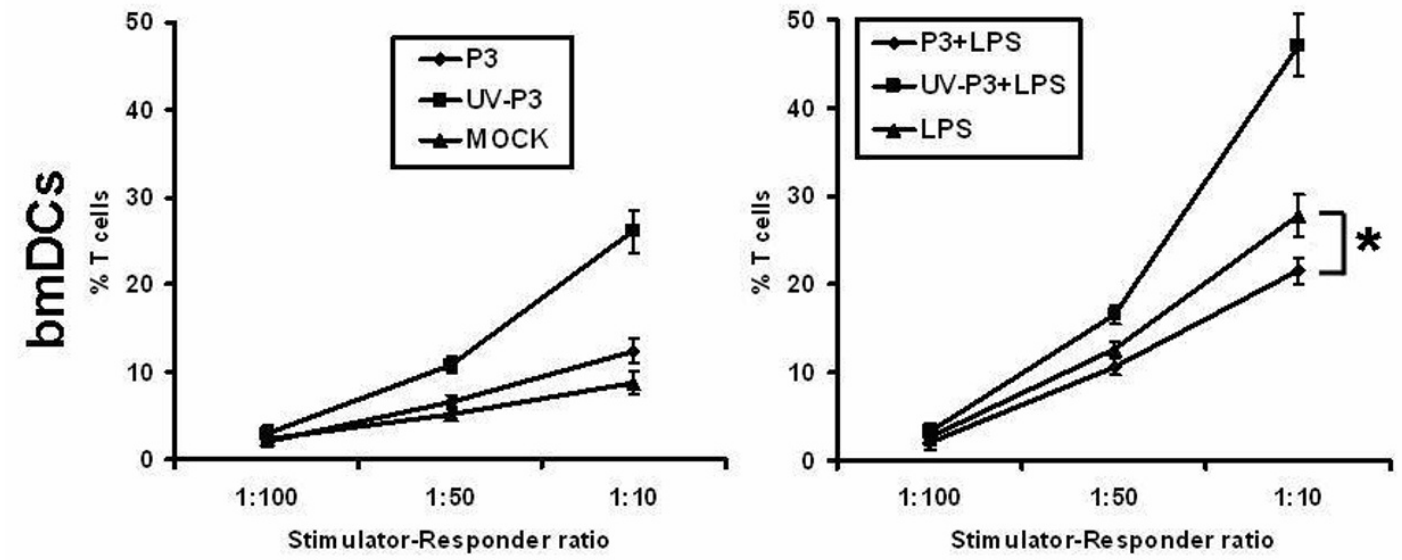

(B)
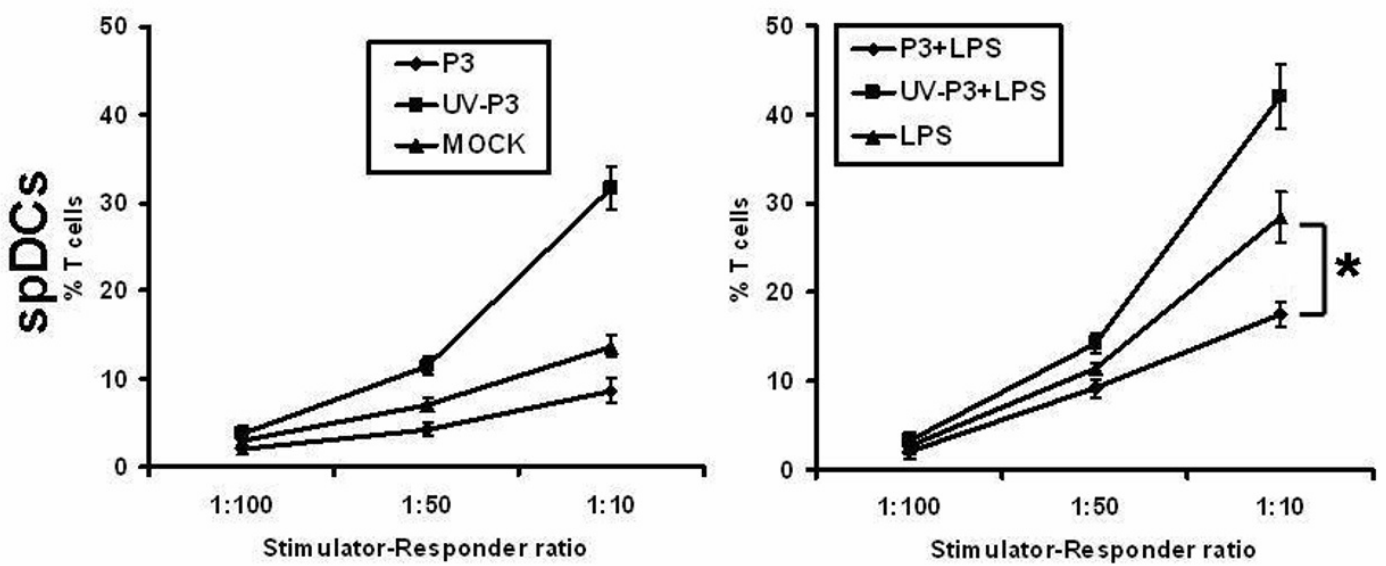

Figure 4 Effects of P3 infection on DCs activation of naïve T cells by MLR. Mock-treated, P3-infected or UV-P3-stimulated DCs as well as differently treated spDCs were added in grade dose to $1 \times 10^{5}$ allogeneic $T$ cells at the indicated stimulator-responder ratios in triplicate, with (B) or without (A) LPS treatment for $20 \mathrm{~h}$ before the addition of $50 \mu \mathrm{l}$ of CellTiter $96^{\circledR}$ AQ ${ }^{\text {ueous }}$ One Solution Cell Proliferation Assay. The bmDCs, spDCs as well as T cells were served as spontaneous NADH/NADPH releases controls respectively. The presentation activities of differently treated bmDCs were measured as 100\% (OD490DC+T exp-OD490 DC spont-OD490T spont $) /($ OD490T spont). Results were expressed as the means \pm SD of triplicates. ${ }^{*}, P<0.05$.

Treg in vivo. The results also demonstrated the expansion of Treg population after the co-culture of P3infected DCs and T cells. It suggested that JEV infection of DCs might influence the mode of T-cell differentiation. Thus, we assumed that induction and expansion of Treg cells by JEV-infected DCs may be associated with immunosuppression in JEV infection. It has previously been shown that immature DCs induced Treg cells are able to suppress other T-cell responses [29-33]. Furthermore, it has been demonstrated that the increased production of IL-10 played an important role in Treg responses which appeared to contribute to immune dysfunction, accounting for viral persistence and acute tissue damage. Therefore, the up-regulation of IL-10 in P3-infected DCs may partly contribute to the expansion of Treg. Based on these results, we suggest that P3 infection may have led to the expansion of Treg cell population in vivo, which could have been involved in the suppression of anti-JEV immune responses. In addition, it is essential to note that although CD25 is expressed on most regulatory $\mathrm{T}$ cells, it is not specific since it can also be expressed on activated CD4+ T cells 
(A)

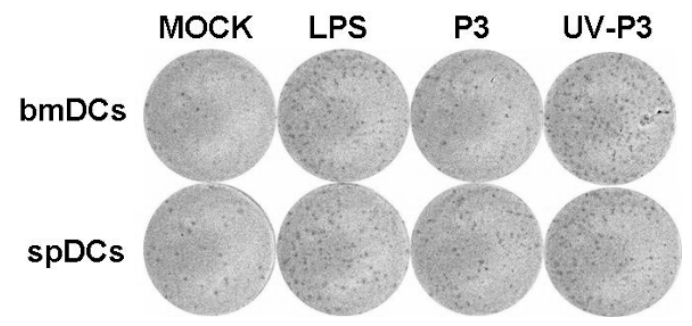

(B)

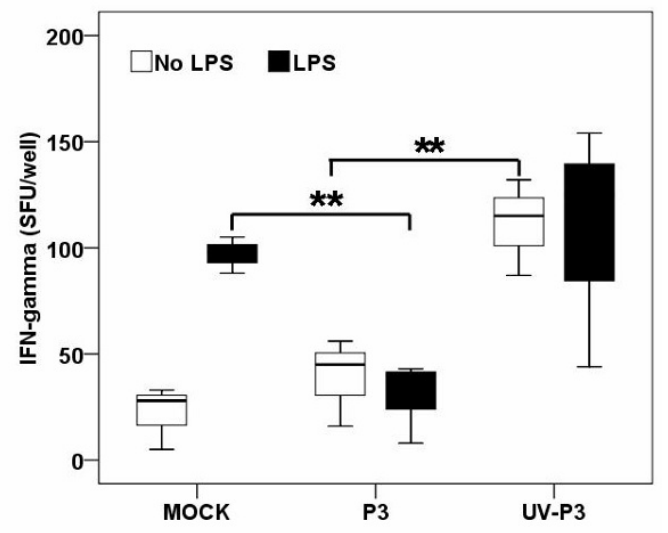

(C)

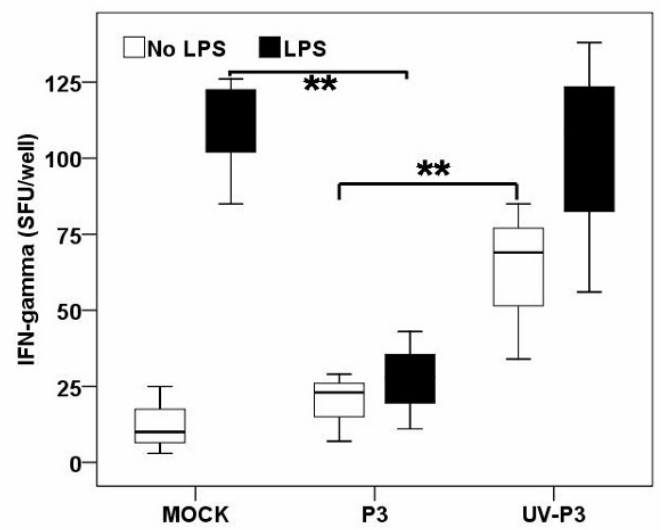

Figure 5 IFN- $\gamma$ producing T cells were detected by ELISPOT assay. P3-infected, UV-P3-stimulated or mock-treated DCs as well as differently treated spDCs were harvested and treated with Mitomycin C (Sigma-Aldrich, MO) at final concentration of $10 \mu \mathrm{g} / \mathrm{ml}$ for $1 \mathrm{~h}$. The differently treated or mock DCs were seeded $\left(1 \times 10^{4}\right.$ per well) together with $1 \times 10^{5}$ per well T cells in triplicates for $20 \mathrm{~h}$. LPSstimulated DC/T cell co-cultures served as positive controls. One representative for IFN- $\gamma$ spot forming unit (SFU) by ELISPOT assay was shown (A). The figure was representative of three independent experiments. Corrected data (SFU)/well were shown for bmDCs and spDCs activations for naïve T cells to expand and produce IFN- $\gamma$ by ELISPOT assay ( $B$, in vitro; $C$, in vivo). Results were expressed as the means \pm SD of triplicates. ${ }^{*}, P<0.05$.

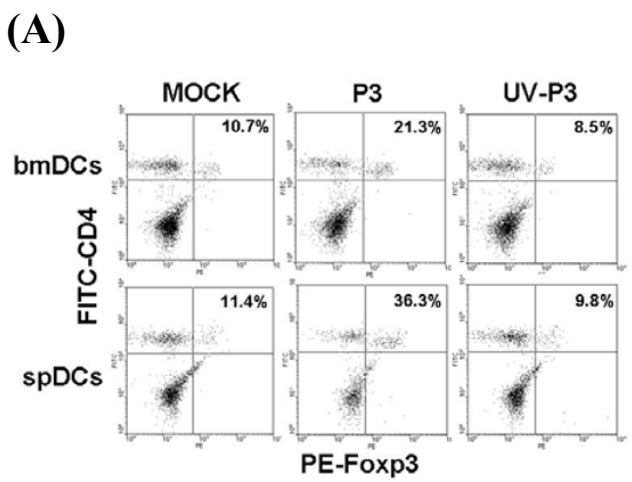

(B)

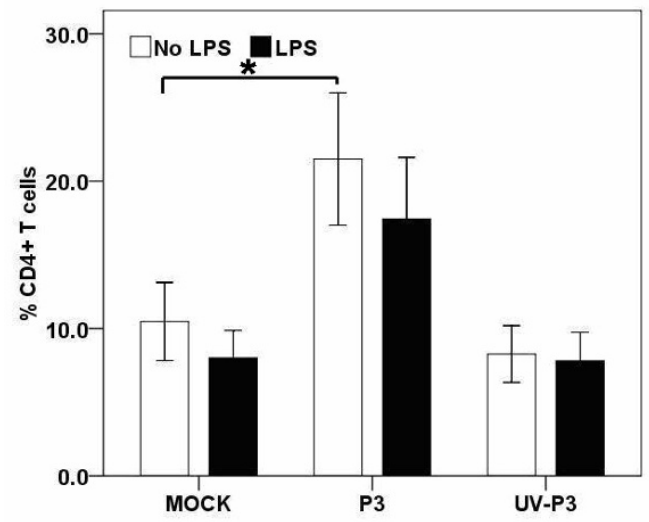

(C)

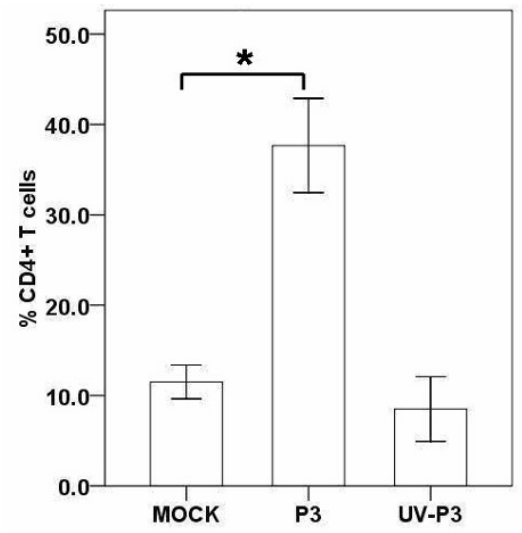

Figure 6 Effects of P3 infection on DCs-induced differentiation of regulatory T cells. $1 \times 10^{5}$ mock-, P3-, UV-P3- or LPS-treated bmDCs were incubated with $1 \times 10^{6}$ allogeneic naïve T cells for 5 days. T cells were purified and doubly labeled for CD4 and Foxp3, and assessed by FACS. The in vivo Treg in splenocytes were purified and examined by FACS from mice inoculated with $1 \times 10^{5}$ PFU P3 or identical UV-P3 i.p. for 5 days. Representative result was shown from three independent experiments (A). The percentage represented the ratio of CD4+ Foxp3+ cells in CD4+ T cells. P3infected bmDCs elicited the Treg differentiation in vitro (B). After P3 infection or UV-P3 stimulation of mice i.p., Treg differentiation in vivo was analyzed immediately (C). Results were expressed as the means \pm SD of triplicates. ${ }^{*}, P<0.05$. 
$[34,35]$. Foxp3 has been shown to be a better marker for CD4+ CD25+ T regulatory cells.

The key cytokines secreted by DCs, including typelIFN (IFN- $\alpha / \beta)$, TNF- $\alpha$, IL-10 and CCL2, restrict the proliferation of invading pathogens and determine the polarization of Th1 and Th2 [36-38]. In particular, secretion of type I IFN is a key step in the innate immune response to viral infection and TNF- $\alpha$ released by DCs can further recruit DC precursors and sustain the antigen presentation [22]. The impaired expression of IFN- $\alpha$ and TNF- $\alpha$ of DCs following the JEV P3 infection when compared with UV-P3 was observed in the present study may contribute to the attenuated generation of antiviral immune response of the host. However, the report of Chang et al., [2005] revealed JEV infection induced IFN- $\beta$ participated in fighting the invading pathogens by using cell types of A549 and SK-N-SH cells through IRF-3- and NF- $\kappa$ Bmediated pathway [39]. Similar results were also obtained in the studies of West nile virus (WNV) infection which induced the IFN- $\alpha$ production of $\mathrm{pDCs}$ and $\mathrm{mDCs}$ [40], while inhibited the IFN- $\beta$ expression of Hela cell [41]. Therefore, we hypothesized that the different cell types from different tissues may present distinct immune response against viral infection. It is known that different cell types usually exert different functions. For instance, pDCs, which generate the crucial signal adaptor IRF7, constitutively express IFN-I. On the contrary, the expression of IFN-I is extremely inhibited in those cell types in absence of the receptor TLR7/TLR9 and IRF-7 [42,43]. Furthermore, different types of cytokines are usually used to discriminate the patterns of immune responses. Therefore, when only considering the individual cell type, different cell types may present distinct immune responses.

TNF- $\alpha$ level in serum and cerebrospinal fluid (CSF) of the fatal case in significantly correlated with prognostic outcome in wild type JEV infection [44]. Therefore, TNF- $\alpha$ may play an important role in immunopathogical responses of the infected host. However, JEV infection of DCs reduced the expression of TNF- $\alpha$ in the current study. On one hand, it usually appears of appropriate expression of TNF- $\alpha$ from the innate response of the host when external pathogen invading. On the other hand, the excess TNF- $\alpha$ induced cell degeneration could be harmful to the survival of virus itself. Therefore, we speculate that the wild type virus may evolve a mechanism by which to restrict the excess inflammatory factors expression at the beginning of the infection, which may facilitate the persistence of the virus survival. Moreover, P3 infection significantly enhanced the release of CCL2 and IL-10. The IL-10 is considered as an anti-inflammatory factor and plays an important role in the differentiation of Treg cells $[31,45,46]$. The suppressed TNF- $\alpha$ production in P3-infected DCs may be partially regulated by high-expressed IL-10. Our results indicated that the release of CCL2 and IL-10 from DCs was positively related to viral infection while the production of IFN- $\alpha$ and TNF- $\alpha$ was negatively related to viral replication. We speculate that the temporary presence of some nonstructure proteins or dsRNA of JEV during the viral replication may play an important role in decelerating or accelerating certain signaling pathway.

Additionally, most data obtained in our experiments are consistent with Aleyas's results except for decreased production of TNF- $\alpha$. This contradicted finding about decreased production of TNF- $\alpha$ might be due to various factors, such as the DCs purity $(>90 \%$ vs $>75 \%)$, JEV strain (P3 and Beijing) and MOI values. All together, the increased level of IL-10 and the decreased productions of IFN- $\alpha$ and TNF- $\alpha$ presented an immunesuppressive profile, indicating the process of the fatal JE development.

\section{Conclusion}

Our data reveals that JEV P3 could infect mouse DCs in vitro and in vivo, and the infection affects the phenotype and function of DCs, including reducing expression of costimulatory molecules, modulating secretion of crucial cytokines, suppressing activation of $\mathrm{T}$ cells, and stimulating differentiation of regulatory $\mathrm{T}$ cells, which indicates that the functional impairment of viral infected DCs orchestrates the immunosuppression in response to the acute JEV infection.

\section{Methods}

\section{Reagents, virus and cells}

The fluorescent antibodies, including CD11c-PE (N418), CD40-FITC (HM40-3), CD80-FITC (16-10A1), CD83FITC (34-1-2S) and MHCl-FITC (Michel-17), recombinant mouse granulocyte-macrophage colony stimulating factor (rmGM-CSF) and IL-4 (rmIL-4) were purchased from eBioscience Inc. (San Diego, CA). The anti-E (JEV envelope protein) MAb was generated in our laboratory and purified with NAb ${ }^{\text {TM }}$ Spin Kits (Thermo Scietific, USA) according to the manufacturer's instructions. JEV P3 strain was produced in BHK-21 which was maintained in Dulbecco's Modified Eagle's Medium (DMEM, Sigma-Aldrich, MO) supplemented with $10 \%$ heated-inactivated fetal bovine serum (FBS, Hyclone, Logan, UT) of $100 \mu \mathrm{g} / \mathrm{ml}$ streptomycin and $100 \mathrm{U} / \mathrm{ml}$ penicillin (Sigma-Aldrich, MO) at $37^{\circ} \mathrm{C}$ with $5 \% \mathrm{CO}_{2}$. And then the virus was tittered by plaque formation assay with BHK-21 cell line. JEV stock was treated with UV irradiation for 1 min (wavelength $253.7 \mathrm{~nm}$, radiation intensity $\geq 60 \mu \mathrm{W} / \mathrm{cm} 2$, distance $30 \mathrm{~cm}$ ).

\section{Generation of bone marrow-derived DCs (bmDCs) and spleen-derived DCs (spDCs)}

For generation of bmDCs from BALB/c mouse bone marrow cultures, the procedure of Inaba et al., [1992] 
was used with minor modifications [47]. Briefly, the bone marrow was flushed from femurs and tibias and subsequently depleted of erythrocytes with ammonium chloride. Cells were plated at $2 \times 10^{6} / \mathrm{ml}$ in DCs media (RPMI 1640 supplemented with 10\% FBS, $100 \mu \mathrm{g} / \mathrm{ml}$ streptomycin, $100 \mathrm{U} / \mathrm{ml}$ penicillin, $10 \mathrm{ng} / \mathrm{ml}$ of $\mathrm{rmGM}$ CSF and rmIL-4). At day 2 and 4 of culturing, 50\% of the supernatant was removed and replenished with fresh DCs media. At day 6, non-adherent cells were collected and transferred into a new dish. After a total of 7 to 9 days of culturing, bmDCs were harvested and purified with StemSep ${ }^{\mathrm{TM}}$ Mouse Dendritic Cell Enrichment Kit (StemCell, Vancouver, BC, Canada).

Four-week old BALB/c mice were infected with $1 \times$ $10^{5}$ PFU of JEV P3 i.p., stimulated with identical quantity of UV-P3 or left mock-treated for 5 days. The splenocytes were obtained from P3-infected or UV-P3stimulated or mock-treated mice. The spDCs were isolated from the splenocytes and purified with StemSep ${ }^{\mathrm{TM}}$ Mouse Dendritic Cell Enrichment Kit (StemCell, Vancouver, BC, Canada) according to the manufacturer's guidelines. The purity of the bmDCs and spDCs fraction was higher than $90 \%$ as determined by FACS analysis of CD11c. Dendritic morphology was assessed by phasecontrast microscopy and viability was assessed by trypan blue exclusion.

\section{JEV P3 infection of DCs}

The immature bmDCs were infected with $\mathrm{P} 3$ at an MOI of 1 . After $1 \mathrm{~h}$ of infection in incomplete medium (DCs media without FBS), cells were washed thoroughly three times and cultured in DCs medium. In some instances, the infected bmDCs were cultured for up to 5 days and on each day cell supernatants were collected and measured for viral RNA quantity. Similarly, the spDCs were harvested from mouse splenocytes every other day thrice after challenge with $10^{5} \mathrm{PFU}$ of JEV per mouse i.p. to detect the viral load in spDCs. Relative levels of viral load in P3-infected bmDCs or spDCs were determined by conducting quantitative real-time PCR analysis by ABI prism 7500 Sequence Detection System (Applied Biosystems) reverse transcription of total RNA isolated from infected samples. Thermal cycling conditions were $2 \mathrm{~min}$ at $50^{\circ} \mathrm{C}, 10 \mathrm{~min}$ at $94^{\circ} \mathrm{C}, 40$ cycles of $15 \mathrm{~s}$ at $94^{\circ} \mathrm{C}$ and then $1 \mathrm{~min}$ at $60^{\circ} \mathrm{C}$. Gene expression was measured by relative quantity and normalized to $\beta$-actin expression by the subtraction of Ct's to provide $\Delta \mathrm{Ct}$ values. After 3 days culture, cells were harvested and used to detect the viral production by RT-PCR and Western blotting and the samples were subjected to PCR. The consensus primers 5'-GCTCTGAAAGGCACAACC-3' (primer1) and 5'-CTGAAGGCATCACCAAAC-3' (primer2) were used to amplify the 467-bp DNA products which were specific for JEV. For Western blotting analysis, cells were collected after 3 days infection and the total proteins were separated by $10 \%$ SDS-PAGE. Separated proteins were electroblotted onto a nitrocellulose membrane. The nonspecific antibody-binding sites were blocked with $1 \%$ bovine serum albumin (BSA) in TBS-T buffer (10 mM Tris- $\mathrm{HCl} \mathrm{pH} 8.0,150 \mathrm{mM} \mathrm{NaCl}$, and $0.05 \%$ Tween-20), and then membranes reacted with anti-E MAb. The resulting blot was treated with peroxidase-conjugated goat anti-mouse IgG (SouthernBiotech, USA). 3, 3-Diaminobenzidine tetrahydrochloride (DAB) was used as substrate for membrane development. The in intro bmDCs were harvested after 3 days infection and the in vivo spDCs were isolated from mice which had been challenged for 5 days. $1 \times$ $10^{5} \mathrm{bmDCs}$ or spDCs were doubly stained with $1.0 \mu \mathrm{g}$ FITC-anti-E and $1.0 \mu \mathrm{g}$ PE-anti-CD11c and analyzed by FACS respectively.

\section{Phenotypic analysis}

After 3 days in vitro infection or 5 days post innoculation, as described in the JEV P3 infection of DCs, the expression of maturation markers of bmDCs and spDCs were determined by FACS on a FACSCalibur (BecktonDickinson [BD], San Jose, CA). $1 \times 10^{5} \mathrm{bmDCs}$ or spDCs were stained with surface marker antibodies including $\mathrm{CD} 11 \mathrm{c}, \mathrm{CD} 40, \mathrm{CD} 80, \mathrm{CD} 83$ and $\mathrm{MHCl}$, or isotype controls at $4^{\circ} \mathrm{C}$ for $30 \mathrm{~min}$ as per manufacturer's guidelines (eBioscience Inc., San Diego, CA). After washing three times with PBS containing 1\% FBS, DCs were phenotypically analyzed by FACS.

\section{Analysis of cytokine production}

The cytokine releases (IFN- $\alpha$, TNF- $\alpha$, CCL2 and IL-10) from P3-infected, UV-P3-stimulated or mock-treated bmDCs or spDCs from differently treated mice were measured by enzyme-linked immunosorbent assay (ELISA) kits (eBioscience Inc., San Diego, CA) in accordance with the manufacturer's guidelines. LPS or poly (IC) served as positive agonist. The concentrations of cytokines in the samples were accessed from the standard curves.

\section{T cells activation capacity of P3-infected DCs (MLR and ELISPOT assay)}

Mixed lymphocyte reactions (MLR) were performed by co-incubation of $1 \times 10^{3}, 2 \times 10^{3}$ or $1 \times 10^{4}$ P3-infected, UV-P3-stimulated or mock-treated, bmDCs or spDCs from differently treated mice with or without $1 \mu \mathrm{g} / \mathrm{ml}$ LPS treatment and $1 \times 10^{5}$ allogeneic naive $\mathrm{T}$ cell per well in 96-well plates (Costar, Cambridge, MA). The mock-treated, P3-infected, UV-P3-stimulated, bmDCs and spDCs or T cells served as spontaneous NADH/ NADPH release controls respectively. After 3 days of incubation in a humidified chamber at $37^{\circ} \mathrm{C}$ in $5 \% \mathrm{CO}_{2}$, 
$50 \mu \mathrm{l}$ of CellTiter $96^{\circledR} \mathrm{AQ}_{\text {ueous }}$ One Solution Cell Proliferation Assay (Promega, Madison, WI, USA) was added to each well for $30 \mathrm{~min}$ at RT, and then $50 \mu \mathrm{l}$ of stop solution (10\% SDS) was added. The absorbance at 490 nm was recorded by ELISA reader (AD340; Beckman Coulter, Fullerton, CA, USA). The activities for activating $\mathrm{T}$ cells of differently treated bmDCs were measured as $100 \%\left(\right.$ OD $490_{\text {DC+T exp. }}-$ OD $490_{\text {DC spont. }}$-OD $\left.490_{\text {T spont. }}\right) /$ (OD490 ${ }_{\mathrm{T} \text { spont. }}$ ).

P3-infected, UV-P3-stimulated or mock-treated bmDCs or spDCs from differently treated mice were harvested and treated with Mitomycin C (SigmaAldrich, MO) at final concentration of $10 \mu \mathrm{g} / \mathrm{ml}$ for $1 \mathrm{~h}$ and washed twice before assessment with enzyme-linked immunospot assay with Mouse IFN- $\gamma$ ELISPOT Kit (eBioscience Inc., San Diego, CA). PVDF-membranebottomed 96-well plates (Millipore) were coated with $10 \mu \mathrm{g} / \mathrm{ml}$ of $\mathrm{mAb}$ on IFN- $\gamma$ in carbonate coating buffer. The treated or mock bmDCs were seeded in triplicates $\left(1 \times 10^{4}\right.$ per well) together with $1 \times 10^{5}$ per well $\mathrm{T}$ cells. LPS (lipopolysacchide, Sigma-Aldrich, MO)-stimulated $\mathrm{DC} / \mathrm{T}$ cell co-cultures were used as controls. After incubation for $20 \mathrm{~h}$, cells were discarded and the plates were washed in PBS-0.05\% Tween and incubated with biotinylated anti-IFN- $\gamma$ mAb (1:1000). After washing, plates were incubated with HRP-Avidin, washed and incubated with AEC solution (Sigma-Aldrich, MO). The staining was stopped by rinsing with water and a red spot was counted as single spot forming unit (SFU). After rewashing, the cytokine-producing cells were visualized with substrate in accordance with the manufacturer's guidelines and counted with an automated ELISPOT reader (AID). The spot-forming T cell number was calculated as following: No. ${ }_{\mathrm{DC}+\mathrm{T}}-\mathrm{No}_{\mathrm{DC}}$

\section{$T$ cell isolation and Treg differentiation}

$\mathrm{T}$ cells from splenocytes of BALB/c mice were enriched by StemSep ${ }^{\mathrm{TM}}$ Mouse T Cell Enrichment Kit (StemCell, Vancouver, BC, Canada) in accordance with the manufacturer's guidelines. Purified T cells were cultured in RPMI 1640 supplemented with 5\% FBS, $1 \times$ nonessential amino acids, $2 \mathrm{mM}$ L-glutamine, $10 \mathrm{mM}$ HEPES, $1 \mathrm{mM}$ sodium pyruvate, $500 \mathrm{nM} 2-\mathrm{ME}, 100 \mu \mathrm{g} / \mathrm{ml}$ streptomycin and $100 \mathrm{U} / \mathrm{ml}$ penicillin.

To assess the impact of JEV infection on Treg cell differentiation in vivo, $1 \times 10^{5}$, P3-infected, UV-P3-stimulated, LPS- or mock-treated bmDCs were added to $1 \times$ $10^{6}$ allogeneic naïve $\mathrm{T}$ cells in 12-well flat-bottom plates (Costar, Cambridge, MA) in triplicate. After 5 days of co-culture, in vitro Treg cells (CD4+ and Foxp3+) were isolated (StemCell, Vancouver, BC, Canada) and stained with Mouse Regulatory T Cell Staining Kit (eBioscience Inc., San Diego, CA) in accordance with the manufacturer's instructions and analyzed by FACS. The in vivo
Treg in splenocytes were purified and conducted on FACS from mice challenged with $10^{5}$ PFU P3 or inoculated with identical UV-P3 for 5 days or from mocktreated mice.

\section{Statistical analysis}

Statistical analysis was performed using the Student's $t$-test. Means were considered significantly different at $P<0.05$.

\section{Acknowledgements}

The authors thank Wanjiku Kagira-Kargbo for her comments on the manuscript modification. This work was supported by the 973 Project of China (No. 2010CB530100), National Natural Sciences Foundation of China (No. 30600446), Transregional Collaborative Research Centre TRR 60 and PCSIRT (IRT0726)

\section{Author details}

${ }^{1}$ State Key Laboratory of Agricultural Microbiology, Huazhong Agricultural University, Wuhan, Hubei 430070, PR China. 'Laboratory of Animal Virology, College of Veterinary Medicine, Huazhong Agricultural University, Wuhan, Hubei 430070, PR China. ${ }^{3}$ College of fisheries, Huazhong Agricultural University, Wuhan, Hubei 430070, PR China.

\section{Authors' contributions}

SC, YL and JY carried out most of the experiments and wrote the manuscript. XY, LC and XL participated part of experiments. HC and SC conceived of the study, participated in its design and coordination, and revised the manuscript. All authors read and approved the final manuscript.

\section{Competing interests}

The authors declare that they have no competing interests.

Received: 26 October 2010 Accepted: 26 January 2011

Published: 26 January 2011

\section{References}

1. Hanna JN, Ritchie SA, Phillips DA, Shield J, Bailey MC, Mackenzie JS, Poidinger M, McCall BJ, Mills PJ: An outbreak of Japanese encephalitis in the Torres Strait, Australia, 1995. Med J Aust 1996, 165:256-260.

2. Sugamata M, Ahmed A, Miura T, Takasu T, Kono R, Ogata T, KimuraKuroda J, Yasui K: Seroepidemiological study of infection with West Nile virus in Karachi, Pakinstan, in 1983 and 1985. J Med Virol 1988, 26:243-247.

3. Khanna N, Agnihotri M, Mathur A, Chaturvedi UC: Neutrophil chemotactic factor produced by Japanese encephalitis virus stimulated macrophages. Clin Exp Immunol 1991, 86:299-303.

4. Ravi V, Parida S, Desai A, Chandramuki A, Gourie-Devi M, Grau GE: Correlation of tumor necrosis factor levels in the serum and cerebrospinal fluid with clinical outcome in Japanese encephalitis patients. J Med Virol 1997, 51:132-136.

5. Singh A, Kulshreshtha R, Mathur A: Secretion of the chemokine interleukin-8 during Japanese encephalitis virus infection. J Med Microbiol 2000, 49:607-612.

6. Ghoshal A, Das S, Ghosh S, Mishra MK, Sharma V, Koli P, Sen E, Basu A: Proinflammatory mediators released by activated microglia induces neuronal death in Japanese encephalitis. Glia 2007, 55:483-496.

7. Balkow S, Krux F, Loser K, Becker JU, Grabbe S, Dittmer U: Friend retrovirus infection of myeloid dendritic cells impairs maturation, prolongs contact to naïve T cells, and favors expansion of regulatory T cells. Blood 2007, 110:3949-3958

8. Moutaftsi M, Mehl AM, Borysiewicz LK, Tabi Z: Human cytomegalovirus inhibits maturation and impairs function of monocyte-derived dendritic cells. Blood 2002, 99:2913-2921.

9. Navas MC, Fuchs A, Schvoerer E, Bohbot A, Aubertin AM, Stoll-Keller F: Dendritic cell susceptibility to hepatitis $C$ virus genotype 1 infection. J Med Virol 2002, 67:152-161. 
10. Se'ne'chal B, Boruchov AM, Reagan JL, Hart DNJ, Young JW: Infection of mature monocyte-derived dendritic cells with human cytomegalovirus inhibits stimulation of T-cell proliferation via the release of soluble CD83. Blood 2004, 103:4207-4215.

11. Beck K, Meyer-König U, Weidmann M, Nern C, Hufert FT: Human cytomegalovirus impairs dendritic cell function: a novel mechanism of human cytomegalovirus immune escape. Eur J Immunol 2003, 33:1528-1538.

12. Knight SC, Patterson S: Bone marrow-derived dendritic cells, infection with human immunodeficient immunodeficiency virus, and immunopathology. Annu Rev Immunol 1997, 15:593-615.

13. Ho LJ, Wang JJ, Shaio MF, Kao CL, Chang DM, Han SW, Lai JH: Infection of human dendritic cells by dengue virus causes cell maturation and cytokine production. J Immunol 2001, 166:1499-1506.

14. Carrasco CP, Rigden RC, Vincent IE, Balmelli C, Ceppi M, Bauhofer O, Tâche V, Hjertner B, McNeilly F, van Gennip HG, McCullough KC, Summerfield A: Interaction of classical swine fever virus with dendritic cells. J Gen Virol 2004, 85:1633-1641.

15. Barba-Spaeth $\mathrm{G}$, Longman RS, Albert ML, Rice CM: Live attenuated yellow fever 17D infects human DCs and allows for presentation of endogenous and recombinant T cell epitopes. J Exp Med 2005, 202:1179-1184.

16. Aleyas AG, George JA, Han YW, Rahman MM, Kim SJ, Han SB, Kim BS, Kim K, Eo SK: Functional modulation of dendritic cells and macrophages by Japanese Encephalitis virus through MyD88 adaptor moleculedependent and -independent pathways. J Immunol 2009, 183:2462-2474.

17. Banchereau J, Steinman RM: Dendritic cells and the control of immunity. Nat Immunol 1998, 392:245-252.

18. Quagliarello VJ, Wispelwey B, Long WJ, Scheld WM: Recombinant human interleukin-1 induces meningitis and blood-brain barrier injury in the rat: characterization and comparison with tumor necrosis factor. J Clin Invest 1991, 87:1360-1366.

19. Li YM, Ye J, Yang XH, Xu M, Chen L, Mei L, Zhu JH, Liu XQ, Chen HC, Cao SB: Japanese encephalitis virus induces cells maturation and triggers T cells activation. Vaccine 2011, 29:855-862.

20. Salio M, Cella M, Suter M, Lanzavecchia A: Inhibition of dendritic cell maturation by herpes simplex virus. Eur J Immunol 1999, 29:3245-3253.

21. Kanto T, Hayashi N, Takehara T, Tatsumi T, Kuzushita N, Ito A, Sasaki Y, Kasahara A, Hori M: Impaired allostimulatory capacity of peripheral blood dendritic cells recovered from hepatitis $C$ virus-infected individuals. J Immunol 1999, 162:5584-5591.

22. Donaghy H, Gazzard B, Gotch F, Patterson S: Dysfunction and infection of freshly isolated blood myeloid and plasmacytoid dendritic cells in patients infected with HIV-1. Blood 2003, 101:4505-4511.

23. Mahanty $S$, Hutchinson $K$, Agarwal S, McRae M, Rollin PE, Pulendran B: Cutting edge: impairment of dendritic cells and adaptive immunity by Ebola and Lassa viruses. J Immunol 2003, 170:2797-2801.

24. Iwashiro M, Messer RJ, Peterson KE, Stromnes IM, Sugie T, Hasenkrug KJ: Immunosuppression by CD4+ regulatory T cells induced by chronic retroviral infection. Proc Natl Acad Sci USA 2001, 98:9226-9230.

25. Ndhlovu LC, Loo CP, Spotts G, Nixon DF, Hecht FM: FOXP3 expressing CD127lo CD4+ T cells inversely correlate with CD38+ CD8+ T cell activation levels in primary HIV-1 infection. J Leukoc Biol 2008, 83:254-262.

26. Dittmer $U$, He H, Messer RJ, Schimmer S, Olbrich AR, Ohlen C Greenberg PD, Stromnes IM, Iwashiro M, Sakaguchi S, Evans LH, Peterson KE, Yang G, Hasenkrug KJ: Functional impairment of CD8+ T cells by regulatory $\mathrm{T}$ cells during persistent retroviral infection. Immunity 2004, 20:293-303.

27. Belkaid $Y$, Rouse BT: Natural regulatory $T$ cells in infectious disease. Nat Immunol 2005, 6:353-360.

28. Li S, Gowans EJ, Chougnet C, Plebanski M, Dittmer U: Natural regulatory T cells and persistent viral infection. J Virol 2008, 82:21-30.

29. Jonuleit H, Schmitt E, Schuler G, Knop J, Enk AH: Induction of interleukin 10-producing, nonproliferating CD4(+) T cells with regulatory properties by repetitive stimulation with allogeneic immature human dendritic cells. J Exp Med 2000, 192:1213-1222.

30. Tarbell KV, Yamazaki S, Steinman RM: The inter-actions of dendritic cells with antigen-specific, regulatory $T$ cells that suppress autoimmunity. Semin Immunol 2006, 18:93-102

31. Yamazaki S, Patel M, Harper A, Bonito A, Fukuyama H, Pack M, Tarbell KV, Talmor M, Ravetch JV, Kayo Inaba, Steinman RM: Effective expansion of alloantigen-specific Foxp3+ CD25+ CD4+ regulatory T cells by dendritic cells during the mixed leukocyte reaction. Proc Natl Acad Sci USA 2006, 103:2758-2763.

32. Gad M, Kristensen NN, Kury E, Claesson MH: Characterization of Tregulatory cells, induced by immature dendritic cells, which inhibit enteroanti-gen-reactive colitis-inducing T-cell responses in vitro and in vivo. Immunology 2004, 113:499-508.

33. Levings MK, Gregori S, Tresoldi E, Cazzaniga S, Bonini C, Roncarolo MG: Differentiation of $\operatorname{Tr} 1$ cells by immature dendritic cells requires IL-10 but not CD25+CD4+ Tr cells. Blood 2005, 105:1162-1169.

34. Fontenot JD, Gavin MA, Rudensky AY: Foxp3 programs the development and function of CD4+ CD25+ regulatory T cells. Nat Immunol 2003, 4:330-336.

35. Morgan ME, van Bilsen JH, Bakker AM, Heemskerk B, Schilham MW, Hartgers FC, Elferink BG, van der Zanden L, de Vries RR, Huizinga TW, Ottenhoff TH, Toes RE: Expression of FOXP3 mRNA is not confined to CD4+ CD25+ T regulatory cells in humans. Hum Immunol 2005, 66:13-20

36. Hilkens $C M$, Kalinski $P$, de Boer $M$, Kapsenberg ML: Human dendritic cells require exogenous interleukin-12-inducing factors to direct the development of naive T-helper cells toward the Th1 phenotype. Blood 1997, 90:1920-1926.

37. Macatonia SE, Hosken NA, Litton M, Vieira P, Hsieh CS, Culpepper JA, Wysocka M, Trinchieri G, Murphy KM, O'Garra A: Dendritic cells produce IL12 and direct the development of Th1 cells from naive CD4+ T cells. J Immunol 1995, 154:5071-5079.

38. Trinchieri G, Scott P: The role of interleukin 12 in the immune response, disease and therapy. Immunol Today 1994, 15:460-463.

39. Chang $T H$, Liao CL, Lin YL: Flavivirus induces interferon-beta gene expression through a pathway involving RIG-I-dependent IRF-3 and PI3K-dependent NF-KB activation. Microbes and Infection 2005, 8:157-171.

40. Silva MC, Guerrero-Plata A, Gilfoy FD, Garofalo RP, Mason PW: Differential activation of human Monocyte-derived and plasmacytoid dentridic cells by West Nile Virus generated in different host cells. J Virol 2007, 81:40-48.

41. Wilson JR, Paola Florez de Sessions, Leon MA, Scholle F: West Nile Virus nonstructural protein 1 inhibits TLR3 signal transduction. J Virol 2008, 82:62-71.

42. Kawai T, Sato S, Ishii KJ, Coban C, Hemmi H, Yamamoto M, Terai K, Matsuda M, Inoue J, Uematsu S, Takeuchi O, Akira S: Interferon-alpha induction through Toll-like receptors involves a direct interaction of IRF7 with MyD88 and TRAF6. Nat Immunol 2004, 5:1061-1068.

43. Akira S: TLR signaling. Microbiol Immunol 2006, 311:1-16.

44. Ravi V, Parida S, Desai A, Chandramuki A, Gourie-Devi M, Grau GE: Correlation of tumor necrosis factor levels in the serum and cerebrospinal fluid with clinical outcome in Japanese encephalitis patients. J Med Virol 1997, 51:132-136.

45. Ng WF, Duggan PJ, Ponchel F, Matarese G, Lombardi G, Edwards AD, Isaacs JD, Lechler RI: Human CD4+ CD25+ cells: a naturally occurring population of regulatory T cells. Blood 2001, 98:2736-2744.

46. O'Garra A, Vieira P: Regulatory T cells and mechanisms of immune system control. Nat Med 2004, 10:801-805.

47. Inaba K, Inaba M, Romani N, Aya H, Deguchi M, Ikehara S, Muramatsu S, Steimman RF: Generation of large numbers of dendritic cells from mouse bone marrow cultures supplemented with granulocyte/macrophage colony-stimulating factor. J Exp Med 1992, 176:1693-1702.

doi:10.1186/1743-422X-8-39

Cite this article as: Cao et al:: Japanese Encephalitis Virus wild strain infection suppresses dendritic cells maturation and function, and causes the expansion of regulatory T cells. Virology Journal 2011 8:39. 